\title{
CONSUMO E COMPORTAMENTO INGESTIVO DE OVINOS MESTIÇOS ALIMENTADOS COM TORTA DO BABAÇU (Orbignya spp.)
}

\author{
INTAKE AND INGESTIVE BEHAVIOR OF CROSSBRED LAMBS FED WITH \\ BABASSU CAKE (Orbignya spp.)
}

\begin{abstract}
Hemilly Cristina Menezes de SÁ $^{1}$; Iran BORGES ${ }^{2}$; Gilberto de Lima MACEDO JUNIOR ${ }^{\mathbf{3}}$; José Neuman Miranda NEIVA ${ }^{4}$; Jhone Tallison Lira de SOUSA ${ }^{5}$; Samara Martins de PAULA ${ }^{5}$

1. Doutoranda, Universidade Federal de Minas Gerais, Escola de veterinária, Departamento de Zootecnia, Belo Horizonte, MG. hemilly.mg@ hotmail.com; 2. Professor associado, Universidade Federal de Minas Gerais, Bolsista de produtividade do CNPq, Belo Horizonte, MG, Brasil; 3. Professor, Doutor, Faculdade de Medicina Veterinária e Zootecnia, Universidade Federal de Uberlândia, Uberlândia, MG, Brasil. gilbertomacedojr@gmail.com; 4. Departamento de Medicina Veterinária e Zootecnia da Universidade Federal de Tocantins, TO, Brasil, Bolsista de produtividade do CNPq. 5. Mestranda em Zootecnia pela Universidade Federal de Tocantins, TO, Brasil.
\end{abstract}

\begin{abstract}
RESUMO: Objetivou-se avaliar o efeito de diferentes inclusões da torta do babaçu sobre o consumo e comportamento ingestivo de ovinos. Vinte ovinos machos não castrados foram distribuídos em delineamento inteiramente ao acaso em quatro tratamentos com cinco repetições por tratamento. Os animais receberam dietas experimentais isofibrosas, isoenergéticas e isonitrogenadas contendo o subproduto em teores crescentes (zero; 7,5\%; 15\%; 22,5\%). Não houve diferença estatística, para os dados de consumo de matéria seca. O consumo de fibra em detergente neutro em porcentagem do peso vivo e gramas por peso metabólico dos animais apresentaram um efeito quadrático obtemos os pontos máximos de $18,00 \%$ e $12,52 \%$ respectivamente de inclusão da torta do babaçu na dieta destes animais. Não se observou diferença significativa entre a eficiência na alimentação pelo consumo de matéria seca e a eficiência na alimentação pelo consumo de fibra em detergente neutro. Houve efeito linear decrescente quanto aos tempos de ócio com as maiores inclusões da torta do babaçu em minutos/dia. Em um programa de criação de ovinos, recomenda-se como alternativa alimentar o uso da torta do babaçu até $15 \%$ na dieta total. A fibra em detergente neutro da torta de babaçu aumentao tempo de mastigação e ruminação dos cordeiros.
\end{abstract}

PALAVRAS-CHAVE: Alimentação. Ruminação. Subproduto. Palmae. Processamento. Ócio.

\section{INTRODUÇÃO}

As pesquisas com subprodutos vêm adquirindo importância indiscutível, diante da necessidade de se viabilizar fontes alternativas para alimentação animal. O uso destes resíduos agroindustriais na alimentação animal pode trazer desempenhos satisfatórios na produção de carne ovina.

O babaçu representa uma das palmeiras brasileiras mais importantes, sendo esta pertencente à família Palmae classificada como Attalea speciosa, Orbygnia martiana, Orbygnia speciosa, Orbignya oleifera. O principal produto extraído do babaçu, e que possui valor comercial e industrial, são as amêndoas contidas em seus frutos para a obtenção do óleo.

O processamento industrial do babaçu é composto de várias etapas onde a amêndoa é moída e em seguida transferida para as prensas à temperatura de $110{ }^{\circ} \mathrm{C}$ ocorrendo o cozimento e prensagem da massa, extraindo-se o óleo, em dois tipos um para fins comestíveis e outro para fins industriais (óleo láurico) e o resíduo conhecido com torta do babaçu possui grande potencial para utilização na nutrição animal.

A principal diferença entre a nomenclatura farelo e a torta do babaçu refere-se ao extrato etéreo, sendo que a extração feita por meio de prensagem recebe o nome de torta já a extração do óleo por meio de solvente recebe o nome de farelo, sendo este com menor teor de extrato etéreo.

As diversas condições de alimentação podem influenciar no comportamento ingestivo. Sendo que o conhecimento do mesmo constitui-se uma ferramenta de grande importância na avaliação das dietas, possibilitando ajustar o manejo alimentar dos animais para obtenção de melhor desempenho produtivo.

Forbes (1995), afirma que as avaliações da etologia ingestiva em ambientes controlados fazemse necessária por propiciar o melhor entendimento das respostas animais assim como sua utilização no desenvolvimento de modelos que servem de suporte às pesquisas possibilitando um melhor desempenho produtivo. Por isso, estudos em etologia vêm sendo cada vez mais utilizados no desenvolvimento de modelos que servem de suporte às pesquisas e às formas de manejo. Assim, objetivou-se avaliar o 
comportamento ingestivo de cordeiros alimentados com crescentes inclusões da torta do babaçu para determinação do teor adequado de inclusão.

\section{MATERIAL E MÉTODOS}

O experimento foi conduzido na Universidade Federal do Tocantins, no setor de ovino caprinocultura, da Escola de Medicina Veterinária e Zootecnia campus Araguaína. Foram utilizados 20 cordeiros machos não castrados com o peso corporal médio inicial de $26 \pm 3,8 \mathrm{~kg}$. Os ovinos foram previamente desverminados e alojados em gaiolas de metabolismo com bebedouros, comedouros plásticos e dispositivos apropriados para coleta de urina e fezes.
A composição percentual da torta do babaçu pode ser observada na Tabela 1. As dietas foram balanceadas segundo o NRC (2007) para ganho de peso de $200 \mathrm{~g}$ gramas/dia contendo quatro teores de inclusão da torta do babaçu, zero; 7,5; 15 e 22,5\% sendo as dietas isonitrogenadas, isoenergéticas e isofibrosas. A composição percentual e nutricional das dietas experimentaispodem serobservadas nas Tabelas 2 e 3 respectivamente. A alimentação dos animais foi oferecida em duas refeições diárias (às oito horas e às 16 horas) sendo que a primeira refeição continha $40 \%$ do total diário ofertado, objetivando a otimização do consumo devido ao clima quente.

Tabela 1. Composição bromatológica da torta do babaçu em porcentagem da matéria seca

Item

Matéria seca
Proteína bruta
Matéria minera
Extrato etéreo
Carboidratos totais
Extratos não
itrogenados

FDN

FDA

NIDN

NIDA

PIDN (\%PB)

Celulose

Lignina

NDT
Torta de babaçu

$$
\begin{gathered}
91,64 \\
25,47 \\
4,59 \\
7,85 \\
62,08 \\
10,04 \\
76,27 \\
43,87 \\
1,43 \\
0,30 \\
35,00 \\
32,10 \\
14,48 \\
39,09
\end{gathered}
$$

FDN = Fibra em detergente neutro; FDA = Fibra em detergente ácido; NIDN= nitrogênio insolúvel em detergente neutro; PIDN= proteína insolúvel em detergente neutro; NIDA= nitrogênio insolúvel em detergente acido; PIDA= proteína insolúvel em detergente acido; PIDNn $(\% \mathrm{~PB})=$ proteína insolúvel em detergente neutro em porcentagem da proteína bruta; NDT $=$ nutrientes digestíveis totaisNDT $=($ DVPB x PB $)+($ EE x2,25 $)+(0,98(100-F D N n-P B-M M-E E-1))+0,75(($ FDNn - LIG $)(L I G / F D N n) 0,667)-7$

Tabela 2. Composição percentual dos ingredientes das dietas (\% na MS)

\begin{tabular}{lllll}
\hline & \multicolumn{4}{l}{ Inclusões da torta do babaçu } \\
\cline { 2 - 5 } Ingredientes & $0 \%$ & $7,50 \%$ & $15 \%$ & $22,50 \%$ \\
\hline Silagem de Capim Napier & 44,30 & 35,30 & 26,30 & 17,32 \\
Fubá Milho & 30,80 & 36,71 & 44,60 & 48,58 \\
Farelo de Soja & 15,90 & 11,30 & 6,73 & 2,16 \\
Fosfato & 3,44 & 3,36 & 3,28 & 3,02 \\
Sal Mineral & 3,00 & 3,00 & 3,00 & 3,00 \\
Calcário & 2,60 & 2,81 & 3,02 & 3,23 \\
Torta babaçu & - & 7,50 & 15,00 & 22,50 \\
\hline
\end{tabular}

*Composição sal mineral: Cálcio 135 g, Fósforo 75 g, Flúor $750 \mathrm{mg}$, Cobalto $80 \mathrm{mg}$, Iodo $80 \mathrm{mg}$, Ferro $3428 \mathrm{mg}$, Cobre $300 \mathrm{mg}$, Magnésio 11g, Zinco 4.000 mg, Enxofre 3 g, Selênio 20 mg, Sódio 145 g. 
Tabela 3. Composição química das dietas experimentais

\begin{tabular}{lllll} 
& \multicolumn{4}{l}{ Inclusões da torta de babaçu } \\
\cline { 2 - 5 } Item & $\mathbf{0 \%}$ & $\mathbf{7 , 5 \%}$ & $\mathbf{1 5 \%}$ & $\mathbf{2 2 , 5 \%}$ \\
\hline Matéria Seca, MS & 87,40 & 70,08 & 75,42 & 80,75 \\
Proteína Bruta (\%MS) & 12,12 & 12,26 & 11,89 & 12,16 \\
PIDN (\%PB) & 15,80 & 18,70 & 19,20 & 19,50 \\
PIDA (\%PB) & 6,70 & 7,40 & 7,80 & 8,10 \\
Extrato etéreo (\%MS) & 2,31 & 2,43 & 2,55 & 2,93 \\
Carboidratos totais (\%MS) & 79,00 & 75,00 & 71,00 & 72,30 \\
FDN(\%MS) & 35,20 & 34,75 & 34,30 & 33,85 \\
FDNcp (\%MS) & 30,07 & 31,54 & 31,38 & 30,33 \\
FDA (\%MS) & 19,31 & 21,35 & 23,56 & 24,31 \\
NDT & 60,98 & 60,37 & 60,23 & 60,14 \\
\hline
\end{tabular}

PIDN= proteína insolúvel em detergente neutro; PIDA= proteína insolúvel em detergente ácido, FDN = Fibra em detergente neutro; FNDcp= fibra em detergente neutro corrigida para cinzas e proteína; FDA = Fibra em detergente ácido; NDT= nutrientes digestíveis totais; NDT $=[\mathrm{PBD}+\mathrm{CNFD}+\mathrm{FDNcpD}+(\mathrm{EED} * 2,25)]$

O período de adaptação dos animais às dietas e às gaiolas foi de 15 dias, sendo o período de coleta (experimental) de cinco dias. Os animais foram pesados no início do período de adaptação. Amostras das rações oferecidas e das sobras foram retiradas e pesadas diariamente. Posteriormente, obteve-se uma amostra composta por animal para as sobras e outra composta das rações fornecidas nos cinco dias de coleta. A coleta total de fezes também foi diária. A produção total teve o peso registrado e fez-se a reserva de uma alíquota de $20 \%$ deste peso. Ao final do experimento foi preparada uma amostra composta por animal. Todas as amostras foram embaladas em sacos plásticos individuais e armazenadas a $-10^{\circ} \mathrm{C}$. Para as determinações de matéria seca, cinzas, extrato etéreo, proteína bruta, do material analisado seguiu-se a metodologia proposta por AOAC (1980). Já para a quantificação da FDN, FDA, celulose, hemiceluloses e ligninas, utilizou-se a metodologia proposta por Van Soest et al. (1991). Para estimativa dos carboidratos totais (CHT) foi usada equação proposta por Sniffen et al. (1992), segundo a qual CHT $=100-(\% \mathrm{~PB}+\% \mathrm{EE}$ $+\% \mathrm{MM}) \mathrm{e}$, para estimativa dos carboidratos não fibrosos (CNF), foi usada a equação preconizada por Hall et al. (1999), qual seja, $\mathrm{CNF}=\% \mathrm{CHT}$ \%FDNCcp, sendo FDNcp a porção de FDN corrigida para cinza e proteína. Para o cálculo dos nutrientes digestíveis totais no ensaio de digestibilidade (NDT), utilizou-se equação proposta por Weiss (1999): NDT $=[$ PBD + CNFD + FDNcpD + (EED * 2,25)], onde PBD; CNFD; FDNcpD e EED significam, respectivamente, consumos de PB, CNF, FDN e EE digestíveis, com a FDN corrigida para cinza e proteína.
O comportamento ingestivo foi realizado durante o período de 24 horas, com observações de cinco em cinco minutos sendo as variáveis comportamentais: ócio $(\mathrm{O})$, ruminação $(\mathrm{R}) \mathrm{e}$ alimentação (A) e mastigação (M) havendo a presença de cinco observadores responsáveis pela observação de quatro animais. O tempo de mastigação foi determinado pelo somatório dos tempos despendidos para alimentação e ruminação. A duração média diária desses períodos de atividades foi calculada dividindo-se a duração total de cada atividade (alimentação, ruminação e ócio em $\mathrm{min} / \mathrm{dia}$ ) pelo seu respectivo número de períodos discretos. A observação noturna dos animais foi realizada mediante o uso de iluminação artificial de lâmpadas incandescentes. O galpão foi mantido com iluminação artificial no período da noite, durante todo o período experimental.

Os resultados referentes aos fatores do comportamento ingestivo, obtidos pela metodologia descrita por Bürger et al. (2000), foram obtidos pelas relações:

$$
\begin{aligned}
& \mathrm{TAL}_{\mathrm{MS}}=\mathrm{CMS} / \mathrm{TeAL} \\
& \mathrm{TAL}_{\mathrm{FDN}}=\mathrm{CFDN} / \mathrm{TeAL} \\
& \text { em que: } \mathrm{TAL}_{\mathrm{MS}}(\mathrm{g} \text { MS consumida/h) e }
\end{aligned}
$$
TAL $_{\text {FDN }}$ (g FDN consumida/h) = taxa de alimentação; CMS $(\mathrm{g})$ = consumo diário de matéria seca; CFDN $(\mathrm{g})=$ consumo diário de FDN; TeAL = tempo gasto diariamente em alimentação.

$\mathrm{TRU}_{\mathrm{MS}}=\mathrm{CMS} / \mathrm{TeRU}$

$\mathrm{TRU}_{\mathrm{FDN}}=\mathrm{CFDN} / \mathrm{TeRU}$

em que: $\mathrm{TRU}_{\mathrm{MS}}$ (g MS ruminada/h) e $\mathrm{TRU}_{\mathrm{FDN}}(\mathrm{g}$ FDN ruminada/h) = taxa de ruminação; TeRU $(\mathrm{h} /$ dia $)=$ tempo de ruminação.

$\mathrm{TMT}=\mathrm{TeAL}+\mathrm{TeRU}$ 
em que: $\mathrm{TMT}(\mathrm{min} / \mathrm{dia})=$ tempo de mastigação total.

O delineamento experimental utilizado foi o inteiramente casualizado com quatro tratamentos e cinco repetições por tratamento. Os dados foram submetidos à análise de variância cujo modelo matemático utilizado foi:

$\mathrm{Yij}=\mu+\tau \mathrm{ij}+\varepsilon \mathrm{ij}$

Onde:

Yij = Observações das variáveis correspondentes à repetição $\mathrm{j}$ sob o tratamento de ordem i;

$\mu=$ Média geral das observações;

$\tau \mathrm{ij}=$ Efeito do tratamento de ordem $\mathrm{i}$;

eij = Erro aleatório associado à repetição de ordem $j$ sob o tratamento de ordem $i$.

As médias foram comparadas utilizando-se o Teste SNK, em nível de 5\% de probabilidade. A análise de regressão foi realizada utilizando-se o software SAEG (SAEG, 2007) para permitir a estimativa dos consumos nas diferentes inclusões, compreendidos no intervalo estudado. Foram testados diferentes modelos matemáticos, a partir do procedimento Modelos Pré-definidos usando o procedimento de MARQUAT do SAEG 9.1, para escolha daquele que apresentasse maior significância e maiores coeficientes de regressão. Para a escolha do modelo matemático também foi observado se o mesmo ajustava-se à resposta biológica.

\section{RESULTADOS E DISCUSSÃO}

O consumo é provavelmente o fator mais importante para determinar o desempenho animal e está relacionado ao perfil de nutrientes do alimento que podem ser digeridos. Considera-se que a ingestão de matéria seca pelos animais seja responsável por 60 a $90 \%$ das variações no seu desempenho, enquanto de 10 a $40 \%$ pode ser explicado pela digestibilidade (MERTENS, 1994). No presente trabalho não houve diferença $(\mathrm{P}>0,05)$, para os dados de consumo de matéria seca (CMS) em gramas/dia (g/dia), porcentagem peso vivo (\%/PV) e unidade do tamanho metabólico (g/UTM) (Tabela 4) demonstrando a possibilidade de inclusão do subproduto na dieta de ovinos. Xenofonte et al. (2008) trabalhando com ovinos SPRD alimentados com dietas contendo $0,10,20$ e $30 \%$ de farelo de babaçu observou um efeito linear decrescente $(\mathrm{P}<0,05)$ dos teores de farelo de babaçu sobre o CMS em g/dia.

Não houve diferença $(\mathrm{P}>0,05)$, para os consumos brutos de FDN em g/dia (CFDN). Porém os consumos relativos de FDN (\%PV e g/UTM) dos animais apresentaram um efeito quadrático com as inclusões da torta de babaçu. A torta do babaçu apresenta alto teor de fibra em detergente neutro e lignina $(76,27 \%$ e $14,48 \%$, respectivamente). Alimentos com altos teores de fibra podem limitar o consumo em função do enchimento ruminal, demostrando haver um limite físico para ingestão do subproduto. Utilizando-se das equações de regressão para os consumos relativos de FDN (\%PV e g/UTM) obtemos os pontos máximos de $18,00 \%$ e $12,52 \%$ respectivamente de inclusão da torta do babaçu na dieta destes animais, sugerindo-se, portanto o teor ideal de inclusão do subproduto.

Tabela 4. Consumos de matéria seca (CMS) e consumo de fibra em detergente neutro (CFDN) de ovinos alimentados com inclusões crescentes da torta do babaçu

\section{Inclusões da torta de babaçu}

\begin{tabular}{lcllllll}
\hline Item & $\mathbf{0 \%}$ & $\mathbf{7 , 5 \%}$ & $\mathbf{1 5 \%}$ & $\mathbf{2 2 , 5 \%}$ & $\mathbf{C V}$ & Eq. Regressão & $\mathbf{R}^{\mathbf{2}}$ \\
\hline CMS (g/dia) & 838,3 & 830,8 & 854,6 & 810,3 & 33,60 & $\hat{Y}$ & - \\
CMS (\%/PV) & 3,02 & 3,64 & 3,38 & 3,05 & 37,17 & $\hat{Y}$ & - \\
CMS (g/UTM) & 69,16 & 79,43 & 75,66 & 69,18 & 35,31 & $\hat{Y}$ & - \\
CFDN (g/dia) & 160,4 & 268,5 & 228,6 & 220,1 & 34,90 & $\hat{Y}$ & - \\
CFDN (\%PV) & 0,58 & 1,15 & 0,91 & 0,82 & 38,72 & $\mathrm{Y}=-0,002 X^{2}+0,072 X+0,628$ & 72,3 \\
CFDN (g/UTM) & 13,29 & 25,17 & 20,35 & 18,72 & 38,16 & Y $=-0,06 X^{2}+1,503 X+14,28$ & 72,5 \\
\hline
\end{tabular}

$(\mathrm{g} / \mathrm{dia})=$ gramas/dia; $(\% / \mathrm{PV})=$ porcentagem peso vivo; $(\mathrm{g} / \mathrm{UTM})=$ unidade do tamanho metabólico; CV- Coeficiente de Variação em porcentagem; $\mathrm{R}^{2}$ coeficiente de determinação $\%$

Não houve diferença $(\mathrm{P}>0,05)$ quanto à taxa alimentação em gramas de matéria seca consumida por hora $\left(\mathrm{TAL}_{\mathrm{MS}}\right)$ como pode ser observado na tabela 5, assim como também nãoforam encontradas diferenças $(\mathrm{P}>0,05)$ nataxa alimentação em gramas de fibra em detergente neutro consumida por hora

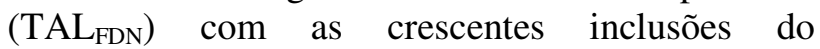
subproduto. 
A ausência de efeitos observada nas taxas de alimentação (TALMS, TALFDN) pode estar relacionada com a ausência de diferenças significativas nos consumos (Tabela 4), assim como a composição química semelhante das dietas (tabela 3), o somatório desses efeitos pode ter contribuído para igualdade dos tratamentos. Carvalho et al. 2008 trabalhando com teores crescentes de farelo de cacau $(0,10,20$ e $30 \%)$ em ovinos, não observou diferenças significativas nas taxas de alimentação e ruminação (g MS e FDN/hora).

Tabela 5. Taxas de alimentação e ruminação de ovinos alimentados com inclusões crescentes da torta do babaçu

\begin{tabular}{|c|c|c|c|c|c|c|c|}
\hline \multicolumn{8}{|c|}{ Inclusões da torta de babaçu } \\
\hline Item & $0 \%$ & $7,5 \%$ & $15 \%$ & $22,5 \%$ & $\mathrm{CV}$ & Eq. Regressão & $\mathbf{R}^{2}$ \\
\hline $\mathrm{AL}_{\mathrm{MS}}$ & 375 & 286,5 & 329 & 264,2 & $\hat{\mathrm{Y}}$ & & 55,3 \\
\hline $\mathrm{TAL}_{\mathrm{FDN}}$ & 71,8 & 92,5 & 87,9 & 71,7 & 45,20 & $\hat{Y}$ & - \\
\hline $\mathrm{TRU}_{\mathrm{MS}}$ & 151,0 & 157,3 & 143,0 & 121,2 & 44,90 & $\hat{Y}$ & - \\
\hline $\mathrm{TRU}_{\mathrm{FDN}}$ & 28,9 & 50,8 & 38,3 & 32,9 & 42,60 & $Y=-0,121 X^{2}+2,721 X+30,97$ & 69 \\
\hline $\mathrm{TMT}_{\mathrm{MS}}$ & 108,0 & 101,5 & 99,8 & 83,1 & 45,20 & $\hat{Y}$ & - \\
\hline $\mathrm{TMT}_{\mathrm{FDN}}$ & 20,6 & 32,8 & 26,7 & 22,5 & 40,90 & $Y=-0,072 X^{2}+1,634 X+21,61$ & 77 \\
\hline
\end{tabular}

CV - Coeficiente de Variação em porcentagem; $\mathrm{R}^{2}$ coeficiente de determinação \% $\mathrm{TAL}_{\mathrm{MS}}=$ alimentação (gramas de matéria seca consumida/hora); $\mathrm{TAL}_{\mathrm{FDN}}=$ taxa alimentação (gramas de fibra em detergente neutro consumida/hora); $\mathrm{TRU}_{\mathrm{MS}}=$ taxa ruminação (gramas de matéria seca consumida/hora); $\mathrm{TRUM}_{\mathrm{FDN}}=$ taxa ruminação gramas de fibra em detergente neutro consumida/hora); $\mathrm{TMT}_{\mathrm{MS}}=$ tempo mastigação, (gramas de matéria seca consumida/hora); $\mathrm{TMT}_{\mathrm{FDN}}=$ tempo mastigação gramas de fibra em detergente neutro consumida/hora).

Segundo Van Soest (1994), o teor de fibra e a forma física da dieta são os principais fatores que afetam a taxa de ruminação. Observamos que TRU $_{\text {FDN }}$ apresentou diferenças $(\mathrm{P}>0,05)$ com a inclusão da torta do babaçu na dieta. Esse resultado indica que os ovinos apresentam mecanismos de adaptação às mudanças dos ingredientes da dieta, já que a torta do babaçu é passível de seleção pelos animais sendo capaz de alterar os consumos relativos de FDN (\%PV e g/UTM) dos animais (Tabela 4) influenciando assim diretamente na

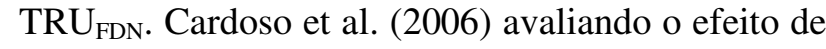
diferentes teores de FDN, na dieta sobre a taxa de alimentação e ruminação da FDN de cordeiros Ile de France $\mathrm{x}$ Texel observaram um comportamento linear crescente, em função da elevação dos teores de fibra das dietas.

A característica fibrosa da torta do babaçu demonstrada nos teores de FDN e lignina na tabela 1 foi capaz de estimular à ruminação $\mathrm{e}$ consequentemente aumentou os valores da tempo mastigação $\left(\mathrm{TMT}_{\mathrm{FDN}}\right)$. Com as inclusões do subproduto nas dietas a quantidade de silagem foi reduzida, porém a efetividade da FDN da torta foi capaz de estimular a mastigação. Dessa forma, pode-se inferir que a FDN da torta de babaçu apresenta características peculiares com relação à efetividade. $\mathrm{TMT}_{\mathrm{FDN}}$ apresentou um efeito quadrático com as crescentes inclusões do subproduto. Segundo, Dado e Allen (1995), o número de períodos de ruminação aumenta de acordo com a qualidade da fibra, o que reflete a necessidade de processamento da digesta ruminal para elevar a eficiência digestiva.

Os resultados relativos aos tempos médios despendidos em alimentação, ócio, ruminação e mastigação minutos por dia, e as respectivas equações de regressão e coeficientes de determinação são apresentados na Tabela 5.

Tabela 5. Comportamento ingestivo das atividades de alimentação, ruminação e ócio e coeficiente de variação (CV) de ovinos alimentados com inclusões crescentes da torta do babaçu

\begin{tabular}{|c|c|c|c|c|c|c|c|}
\hline \multirow[b]{2}{*}{ Item } & \multicolumn{4}{|c|}{ \% Torta de babaçu } & \multirow[b]{2}{*}{$\mathbf{C V}$} & \multirow[b]{2}{*}{ Eq. Regressão } & \multirow[b]{2}{*}{$\mathbf{R}^{2}$} \\
\hline & $\mathbf{0}$ & 7,5 & 15,0 & 22,5 & & & \\
\hline \multicolumn{5}{|c|}{ Tempo gasto minutos } & & & \\
\hline Alimentação & 134,0 & 174,0 & 156,0 & 184,0 & 26,90 & $\hat{Y}$ & - \\
\hline Ócio & 970,0 & 950,0 & 926,0 & 850,0 & 9,20 & $Y=981,6-5,12 X$ & 89,0 \\
\hline Ruminação & 333,0 & 317,0 & 358,0 & 401,0 & 19,75 & $\hat{\mathrm{Y}}$ & - \\
\hline Mastigação & 467,0 & 491,0 & 514,0 & 585,0 & 16,63 & $\mathrm{Y}=457,7+5,026667 \mathrm{X}$ & 87,0 \\
\hline
\end{tabular}

CV - Coeficiente de Variação em porcentagem; $\mathrm{R}^{2}$ coeficiente de determinação \% 
Os tempos de alimentação em minutos não apresentaram diferença estatística $(\mathrm{P}<0,05)$ com as diferentes inclusões da torta do babaçu. $\mathrm{O}$ fato das dietas terem sido isonitrogenadas e isofibrosas assim como a igualdade no consumo de MS pode explicar a semelhança entre os tempos. Carvalho et al. (2008) avaliando o comportamento de ovinos recebendo inclusões crescentes farelo de cacau $(0$, 10,20 e $30 \%$ ) não observaram variações significativa nos tempos de alimentação em minutos. O alto teor de FDN da torta do babaçu classifica-a como um subproduto fibroso (tabela 1), essa característica poderia ter limitado a alimentação pelos animais, porém os tempos de alimentação não apresentaram diferença sugerindo a utilização crescente do subproduto em dietas para ovinos.

Houve efeito linear decrescente $(\mathrm{P}<0,05)$ quanto aos tempos de ócio com as maiores inclusões da torta do babaçu em minutos/dia. Possivelmente explicados pelo fato dos animais gastarem mais tempo mastigando (minutos/dia) com a inclusão da torta de babaçu nas dietas experimentais, pois com a inclusão da torta de babaçu na dieta impreterivelmente a quantidade de volumoso é reduzida em função da característica fibrosa desse alimento. Segundo Dulphy et al. (1980), quando aumentam os constituintes da parede celular da dieta, eleva-se o tempo total de mastigação em minutos/dia, o que pode ser observado na equação de regressão em função da inclusão da torta do
SÁ, H. C. M. et al.

babaçu em teores crescentes apresentando efeito linear positivo. Fimbres et al. (2002) trabalharam com teores crescentes de feno na alimentação de ovinos $(0,10,20$ e $30 \%$ da matéria seca) observaram um efeito linear decrescente $(\mathrm{P}<0,05)$ no tempo de ócio em minutos por dia, com o aumento da quantidade de feno na dieta.

O tempo de mastigação é o somatório do tempo gasto com alimentaçãoe ruminação. Assim, mesmo essas duas variáveis, alimentaçãoe ruminação, não terem apresentado diferença, sua soma alterou o tempo gasto com a mastigação e consequentemente reduziu o tempo em ócio. Gonçalves et al. (2001), trabalhando com cabras leiteiras, alimentadas com dietas com diferentes relações volumoso:concentrado (100:0; 80:20; $60: 40 ; 40: 60$ e 20:80), verificaram que, com o aumento da fibra nas dietas, houve aumento nos tempos despendidos com alimentação e ruminação e, em contrapartida, houve dimin. uição no tempo despendido com ócio.

\section{CONCLUSÕES}

Em um programa de criação de ovinos, recomenda-se como alternativa alimentar o uso da torta do babaçu até $15 \%$ na dieta total.

A fibra em detergente neutro da torta de babaçu aumenta a tempo de mastigação e ruminação dos cordeiros.

\begin{abstract}
The objective was to evaluate the effect of different inclusions of babassu cake over the intake and ingestive behavior of lambs. Twenty male lambs were distributed in a randomized design in four treatments with five repetitions per treatment. The animals received isofiber, isocaloric and isonitrogen diets with increasing levels of babassu cake (zero; $7,5 \% ; 15 \% ; 22,5 \%$ ). There was no statistical difference for the dry matter intake. The neutral detergent fiber intake of percentage of body weight and of grams per metabolic weight presented a quadratic effect which maximum points were $18,00 \%$ and $12,52 \%$ respectively of inclusion of babassu cake in the diet. There was no difference between efficiencies in the feeding of dry matter and neutral detergent fiber intakes. There was a decreasing linear effect for the idling time in minutes/day with the higher inclusions of babassu cake. In a production system of sheep, it is recommended as an alternative feeding the use of babassu cake at $15 \%$ of the diet. The neutral detergent fiber of the babassu cake increases the chewing and ruminating rates of lambs.
\end{abstract}

KEYWORDS: By product. Feeding. Idle. Palmae. Processing. Rumination.

\title{
REFERÊNCIAS
}

AOAC. Association of Official Analytical Chemists. Official Methods of Analysis (red.). Washington DC: AOAC, 1980. 1015p

BÜRGER, P. J.; PEREIRA, J. C.; QUEIROZ, A. C.; COELHO DA SILVA, J. F.; VALADARES FILHO, S. C.; CECON, P. R.; CASALI, A. D. P. Comportamento ingestivo em bezerros holandeses alimentados com dietas contendo diferentes níveis de concentrado. Revista Brasileira de Zootecnia, Viçosa, v. 29, n. 1, p. 236242, Fev. 2000. 
CARdoso, A. R.; CARVAlHO, S.; GALVANI, D. B.; PIRES, C. C.; GASPERIN, B. G.; GARCIA, R. P. A. Comportamento ingestivo de cordeiros alimentados com dietas contendo diferentes níveis de fibra em detergente neutro. Ciência Rural, Santa Maria, v. 36, n. 2, p. 604-609, Mar-Abr, 2006.

http://dx.doi.org/10.1590/S0103-84782006000200038

CARVALHO, G. G. P.; PIRES, A. J. V.; SILVA, R. R.; RIBEIRO, L. S. O.; CHAGAS, D. M. T. Comportamento ingestivo de ovinos Santa Inês alimentados com dietas com farelo de cacau. Revista Brasileira de Zootecnia, Viçosa, v. 37, n. 4, p. 660-665, Abr. 2008.

DADO, R. G.; ALLEN, M. S. Intake limitation, feeding behavior, and rumen function of cows challenged with rumen fill from dietary fiber or inert bulk. Journal of Dairy Science, Champaign, v. 78, n. 1, p. 118-133, Jan. 1995. http://dx.doi.org/10.3168/jds.S0022-0302(95)76622-X

DULPHY, J. P.; REMOND, B.; THERIEZ, M. Ingestive behavior and related activities in ruminants. In: RUCKEBUSH, Y.; THIVEND, P. (Ed.). Digestive physiology and metabolism in ruminants. Lancaster: MTP, 1980. p. 103-122. http://dx.doi.org/10.1007/978-94-011-8067-2_5

FIMBRES, H.; KAWAS, J. R.; HERNADEZ-VIDAL, J. F.; PICÓNRUBIO, C. D.; LU, C. D. Nutrient intake, digestibility, mastication and ruminal fermentation of lambs fed finishing ration with various forage levels.

Small Ruminant Research, Philadelphia, v. 43, n. 3, p. 275-281, Mar. 2002. http://dx.doi.org/10.1016/S09214488(02)00013-5

FORBES, J. M. Voluntary food intake and diet selection in farm animals. 2. ed. Wallingford, CAB International, 1995. 532p.

GONÇALVES, A. L.; LANA, R. P.; RODRIGUES, M. T.; MENDONÇA, R. A.; QUEIROZ, V. A. C.; HENRIQUE, D. S. S. Padrão nictemeral do $\mathrm{pH}$ ruminal e comportamento alimentar de cabras leiteiras alimentadas com dietas contendo diferentes relações volumoso:concentrado. Revista Brasileira de Zootecnia, Viçosa, v. 30, n. 6, p. 1886-1892, Dez. 2001.

HALL, M. B.; HOOVER,W. H.; JENNINGS, J. P. A method for partitioning neutral detergent soluble carbohydrates. Journal Science Food Agriculture, Gainesville, v. 79, p. 2079-2086, 1999.

http://dx.doi.org/10.1002/(SICI)1097-0010(199912)79:15<2079::AID-JSFA502>3.0.CO;2-Z

MERTENS, D. R. Regulation of forage intake. In: FAHEY JR., G. C. (Ed.). Forage quality, evaluation and utilization.Winsconsin: American Society of Agronomy. 1994. p. 450-493.

NRC. Nutrient requirement of sheeps. Washington, D. C.: National Academy Press, 2007.

SNIFFEN, C. J., O'CONNOR, J.D.; VAN SOEST, P.J. et al. A net carbohydrate and protein system for evaluating cattle diets: II. Carbohydrate and protein availability. Journal of Animal Science, Champaign, v. 70, n. 12, p. 3562-3577, 1992.

UFV. 1997. SAEG. Sistema de análises estatísticas e genética (Versão 9.1). Universidade Federal de Viçosa. Viçosa, MG

VAN SOEST, P. J. Nutritional ecology of the ruminant. 2. ed. Ithaca: Cornell University Press, 1994. 476p

VAN SOEST, P. J., ROBERTSON, J. B.; LEWIS, B. A. Symposium: Carbohydrate methodology, metabolism, and nutritional implications in dairy cattle. Journal Dairy Science, Champaign, v. 74, n. 10, p. 3583-3597, 1991. http://dx.doi.org/10.3168/jds.S0022-0302(91)78551-2

WEISS, W. P. Energy prediction equations for ruminant feeds. In: Cornell nutrition conference for feed manufacturers, 61., 1999, Ithaca. Proceedings. Ithaca: Cornell University, 1999.

XENOFONTE, A. R. B.; CARVALHO F. F. R.; BATISTA A. M. V.; MEDEIROS, G. R.; ANDRADE, R. P. $\mathrm{X}$. Desempenho e digestibilidade de nutrientes em ovinos alimentados com rações contendo farelo de babaçu.

Revista Brasileira de Zootecnia, Viçosa, v. 37, n. 11, p. 2063-2068, Nov. 2008. 